\title{
Bijective Enumeration of Bicolored Maps of Given Vertex Degree Distribution
}

\author{
Alejandro Morales $\|^{\mid}$and Ekaterina Vassilieva ${ }^{2}$ \\ ${ }^{1}$ Department of Mathematics, Massachusetts Institute of Technology, Cambridge MA 02139 \\ ${ }^{2}$ Laboratoire d'Informatique de l'Ecole Polytechnique, 91128 Palaiseau Cedex, France
}

\begin{abstract}
We derive a new formula for the number of factorizations of a full cycle into an ordered product of two permutations of given cycle types. For the first time, a purely combinatorial argument involving a bijective description of bicolored maps of specified vertex degree distribution is used. All the previous results in the field rely either partially or totally on a character theoretic approach. The combinatorial proof relies on a new bijection extending the one in [G. Schaeffer and E. Vassilieva. J. Comb. Theory Ser. A, 115(6):903-924, 2008] that focused only on the number of cycles. As a salient ingredient, we introduce the notion of thorn trees of given vertex degree distribution which are recursive planar objects allowing simple description of maps of arbitrary genus.

Résumé. Nous démontrons une nouvelle formule exprimant le nombre de factorisations d'un long cycle en produit de deux permutations ayant un type cyclique donné. Pour la première fois, nous utilisons un argument purement combinatoire basé sur une description bijective des cartes bicolores dont la distribution des degrés des sommets est donnée. Tous les résultats précédents dans le domaine se basent soit partiellement soit totalement sur la théorie des caractères de groupe. La preuve combinatoire se fonde sur une nouvelle bijection généralisant celle introduite dans [G. Schaeffer and E. Vassilieva. J. Comb. Theory Ser. A, 115(6):903-924, 2008] ne s'intéressant qu'au nombre de cycles. L'ingrédient le plus saillant est l'introduction de la notion d'arbre épineux de structure cyclique donnée, des objets récursifs et planaires permettant une description simple des cartes de genus arbitraire.
\end{abstract}

Keywords: bicolored maps, full cycle factorization, vertex degree distribution

\footnotetext{
${ }^{\dagger} \mathrm{AM}$ was supported by the EP/MIT internship program.

1365-8050 @ 2009 Discrete Mathematics and Theoretical Computer Science (DMTCS), Nancy, France
} 


\section{Full cycle factorizations of given type}

In what follows, we denote by $\lambda \vdash n$ an integer partition of $n$ and $\ell(\lambda)=k$ the length or number of parts of $\lambda$. Thus, $\lambda=\left(\lambda_{1}, \ldots, \lambda_{k}\right)$ where $\lambda_{1} \geq \cdots \geq \lambda_{k} \geq 1$ and $\sum \lambda_{i}=n$. If $m_{i}(\lambda)$ is the number of parts of $\lambda$ that are equal to $i$, then we also write $\lambda$ as $\left[1^{m_{1}(\lambda)}, 2^{m_{2}(\lambda)}, \ldots\right]$ and let $A u t(\lambda)=\prod_{i} m_{i}(\lambda)$ !. Then, for $\lambda \vdash n$, we use the monomial symmetric function $m_{\lambda}(x)$ which is the sum of all different monomials obtained by permuting the variables of $x_{1}^{\lambda_{1}} x_{2}^{\lambda_{2}} \cdots$, and the power symmetric function $p_{\lambda}(x)$, defined multiplicatively as $p_{\lambda}=p_{\lambda_{1}} p_{\lambda_{2}} \ldots$ where $p_{n}(x)=m_{n}(x)=\sum_{i} x_{i}^{n}$ (see e.g. (10)).

In addition, we use $\pi$ to denote a set partition of $[n]=\{1,2, \ldots, n\}$ with blocks $\left\{\pi^{1}, \ldots, \pi^{p}\right\}$. The type of a set partition, type $(\pi) \vdash n$, is the integer partition of $n$ obtained by considering the cardinalities of the blocks of $\pi$. Let $S_{n}$ be the symmetric group on $n$ elements, and $\mathcal{C}_{\lambda}$ be the conjugacy class in $S_{n}$ of permutations with cycle type $\lambda$, where $\lambda \vdash n$. Given $\lambda, \mu, \nu \vdash n$, let $c_{\lambda \mu}^{\nu}$ be the number of ordered factorizations in $S_{n}$ of a fixed permutation $\gamma \in \mathcal{C}_{\nu}$ as a product $\alpha \beta$ of two permutations $\alpha \in \mathcal{C}_{\lambda}$ and $\beta \in$ $\mathcal{C}_{\mu}$. These numbers are called connection coefficients of the symmetric group. The problem of computing these coefficients has received significant attention and its history and references can be found in (3). We focus on the case $c_{\lambda \mu}^{n}$ : when $\nu=(n)=n$ and $\gamma$ is the long cycle $\gamma_{n}=(1,2, \ldots, n)$. In this setting, we define the genus $g(\lambda, \mu)$ of a pair of partitions $\lambda$ and $\mu$ by the equation $\ell(\lambda)+\ell(\mu)=n+1-2 g(\lambda, \mu)$. We can take $g(\lambda, \mu)$ to be a nonnegative integer, since otherwise it is easy to show that $c_{\lambda \mu}^{n}=0$.

Regarding the evaluation of $c_{\lambda \mu}^{n}$, using an inductive argument Bédard and Goupil (1) first found a formula for the case $g(\lambda, \mu)=0$, which was later reproved by Goulden and Jackson (2) via a bijection with a set of ordered rooted bicolored trees. Later, using characters of the symmetric group and a combinatorial development, Goupil and Schaeffer (3) derived an expression for connection coefficients of arbitrary genus as a sum of positive terms (see Biane (4) for a succinct algebraic derivation; and Poulalhon and Schaeffer (4), and Irving (7) for further generalizations). However, there are no fully bijective proofs for this expression unless the permutations are associated to set partitions (see Goulden and Nica (6)). In this paper, we follow the latter approach and introduce the notion of partitioned bicolored maps of given type to extend the work of Schaeffer and Vassilieva in (9), and derive a novel simpler formula thanks to a purely combinatorial argument. In the genus zero case, the argument reduces to the bijection with ordered rooted bicolored trees in (2). Our combinatorial result can be stated as follows:

Theorem 1 The numbers $c_{\lambda \mu}^{n}$ of factorizations of the long cycle $\gamma_{n}$ into an ordered product of two permutations of type $\lambda$ and $\mu$ satisfy:

$$
\frac{1}{n} \sum_{\lambda, \mu \vdash n} c_{\lambda \mu}^{n} p_{\lambda}(x) p_{\mu}(y)=\sum_{\lambda, \mu \vdash n} \frac{(n-\ell(\lambda)) !(n-\ell(\mu)) !}{(n+1-\ell(\lambda)-\ell(\mu)) !} m_{\lambda}(x) m_{\mu}(y)
$$

We start in Section 2 by defining the main combinatorial structures used throughout the paper and reformulating Theorem 1. In Section 3 we introduce a mapping among these structures and in Section 4 we show it is a bijection.

\section{Link with maps and partitioned maps of specified type}

\subsection{Unicellular partitioned bicolored maps}

Definition 1 Given partitions $\lambda, \mu \vdash n$, let $\mathcal{C}(\lambda, \mu)$ be the set of triples $\left(\pi_{1}, \pi_{2}, \alpha\right)$ such that $\alpha \in S_{n}$, $\pi_{1}, \pi_{2}$ are set partitions of $[n]$ with type $\left(\pi_{1}\right)=\lambda$ and type $\left(\pi_{2}\right)=\mu$, and each block of $\pi_{1}$ and $\pi_{2}$ is a union of cycles of $\alpha$ and $\beta=\alpha^{-1} \gamma_{n}$ respectively. Let $C(\lambda, \mu)=|\mathcal{C}(\lambda, \mu)|$. 
In accordance with the following graphical interpretation, each factorization $\alpha \beta=\gamma_{n}$ is called a unicellular bicolored map. Similarly, each triple $\left(\pi_{1}, \pi_{2}, \alpha\right)$ is called a unicellular partitioned bicolored map with $n$ edges, blocks of type $\lambda$ consisting of white vertices, and blocks of type $\mu$ consisting of black vertices. We represent these unicellular maps graphically using ribbon graphs:

A ribbon graph is a drawing of a graph in the plane such that any vertex of degree $k$ has a neighborhood homeomorphic to a disk and the incident edges form a star with $k$ branches. Edges can cross outside of these neighborhoods, and we call these irrelevant crossings. Moreover, given an edge $e$ of a ribbon graph we assume it is oriented and identify the right hand side of $e$ in the direction given by the orientation. We describe the boundary of the graph by moving along the edges. In doing so, we ignore irrelevant crossings and when we reach a vertex, we continue in the next branch of the star without crossing any edge in the neighborhood of the vertex. A graph is bicolored if its vertices are colored black and white such that each edge connects a black vertex with a white one. In this case the right hand side of the edge is the side of the edge which is on the right when going from a white vertex to a black one.

Then a unicellular partitioned bicolored map with $n$ edges is represented by a labeled ribbon graph with the following six properties: (i) $n$ edges with the labels $[n]=\{1, \ldots, n\}$, (ii) the cycles of $\alpha$ describe the white vertices, and (iii) the partition $\pi_{1}$ induces a partition of the white vertices of type $\lambda$. Properties (iv), (v), and (vi) are the analogues of (i), (ii), and (iii) for $\beta$, black vertices, $\pi_{2}$ and $\mu$ respectively. The fact that $\alpha \beta=\gamma_{n}$ means that if we start on the right hand side of the edge with label 1 and move along the edges as described above, we visit the right hand side of the edges $1, \ldots, n$ in this order.

Example 1 Let $n=10, \lambda=[4,6], \mu=\left[2^{2}, 6\right], \alpha=(25)(3467)(18910) \in \mathcal{C}_{\left[2,4^{2}\right]}$ and $\beta=\alpha^{-1} \gamma_{n}=$ $(3)(6)(15427)(8)(9)(10) \in \mathcal{C}_{\left[1^{5}, 5\right]}, \pi_{1}=\{3467,1258910\}$, and $\pi_{2}=\{1245710,68,39\}$. We easily check that $g\left(\left[2,4^{2}\right],\left[1^{5}, 5\right]\right)=1$ and $\left(\pi_{1}, \pi_{2}, \alpha\right) \in \mathcal{C}(\lambda, \mu)$. We represent this unicellular partitioned bicolored map with the following ribbon graph with one irrelevant crossing and where each block is associated with a particular shape:

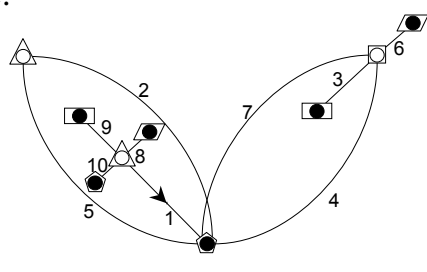

Fig. 1: A Partitioned Bicolored Map

\subsection{Connection between $\mathcal{C}(\lambda, \mu)$ and $c_{\lambda \mu}^{n}$}

Consider the partial order on integer partitions given by refinement. That is $\lambda \preceq \mu$ if and only if the parts of $\mu$ are unions of parts of $\lambda$, and we say that $\lambda$ is a refinement of $\mu$ or that $\mu$ is coarser than $\lambda$. If $\lambda \preceq \mu$ let $\bar{R}_{\lambda \mu}$ be the number of ways to coarse $\lambda$ to obtain $\mu$. For example, if $\lambda=1^{2} 2^{2}$ and $\mu=123$ then $\bar{R}_{\lambda \mu}=4$ since in $\lambda$ any of the two 1-blocks can merge with any of the two 2-blocks. See (11) for more about the poset of integer partitions ordered by refinement.

Remark 1 If $m=\ell(\lambda)$ and $p=\ell(\mu)$ then $\bar{R}_{\lambda \mu}$ is equal to the number of unordered partitions $\pi=$ $\left\{\pi_{1}, \ldots, \pi_{p}\right\}$ of the set $[\mathrm{m}]$ such that $\mu_{j}=\sum_{i \in \pi_{j}} \lambda_{i}$ for $1 \leq j \leq p$. Therefore, for the monomial and 
power symmetric functions, $m_{\lambda}$ and $p_{\lambda}$, we have: $p_{\lambda}=\sum_{\mu \succeq \lambda} R_{\lambda \mu} m_{\mu}$, where $R_{\lambda \mu}=A u t(\mu) \bar{R}_{\lambda \mu}(10$. Prop.7.7.1).

We use this partial order on integer partitions to obtain a relation between $C(\lambda, \mu)$ and $c_{\lambda \mu}^{n}$.

Proposition 1

$$
C(\nu, \rho)=\sum_{\lambda \preceq \nu, \mu \preceq \rho} \bar{R}_{\lambda \nu} \bar{R}_{\mu \rho} c_{\lambda \mu}^{n}
$$

Proof: Let $\left(\pi_{1}, \pi_{2}, \alpha\right) \in \mathcal{C}(\nu, \rho)$. If $\alpha \in \mathcal{C}_{\lambda}$ and $\beta=\alpha^{-1} \gamma_{n} \in \mathcal{C}_{\mu}$ then by definition of the set partitions we have that $\operatorname{type}\left(\pi_{1}\right)=\nu \succeq \lambda$ and $\operatorname{type}\left(\pi_{2}\right)=\rho \succeq \mu$. Thus, if

$$
\mathcal{C}_{\lambda \mu}(\nu, \rho)=\left\{\left(\pi_{1}, \pi_{2}, \alpha\right) \in \mathcal{C}(\mu, \rho) \mid\left(\alpha, \alpha^{-1} \gamma_{n}\right) \in \mathcal{C}_{\lambda} \times \mathcal{C}_{\mu}\right\}
$$

then $\mathcal{C}(\nu, \rho)=\bigcup_{\lambda \preceq \nu, \mu \preceq \rho} \mathcal{C}_{\lambda \mu}(\nu, \rho)$ where the union is disjoint. Finally, if $C_{\lambda \mu}(\nu, \rho)=\left|\mathcal{C}_{\lambda \mu}(\nu, \rho)\right|$ then it is easy to see that $C_{\lambda \mu}(\nu, \rho)=\bar{R}_{\lambda \nu} \bar{R}_{\mu \rho} c_{\lambda \mu}^{n}$.

Using $p_{\lambda}=\sum_{\nu \succeq \lambda} R_{\lambda \nu} m_{\nu}$ we can rewrite Proposition 1 as:

$$
\sum_{\lambda, \mu \vdash n} c_{\lambda \mu}^{n} p_{\lambda}(x) p_{\mu}(y)=\sum_{\lambda, \mu \vdash n} \operatorname{Aut}(\lambda) A u t(\mu) C(\lambda, \mu) m_{\lambda}(x) m_{\mu}(y) .
$$

Remark 2 Let $\left(\pi_{1}, \pi_{2}, \alpha\right) \in \mathcal{C}(\nu, \rho)$ with $\ell(\nu)+\ell(\rho)=n+1$. If $\alpha \in \mathcal{C}_{\lambda}$ and $\beta \in \mathcal{C}_{\mu}$ then $\ell(\lambda)+\ell(\mu)=$ $n+1-2 g(\lambda, \mu) \leq n+1$. But $\ell(\lambda) \geq \ell(\nu)$ and $\ell(\mu) \geq \ell(\rho)$, therefore $\lambda=\nu, \mu=\rho$; and $\pi_{1}$ and $\pi_{2}$ are the underlying set partitions in the cycle decompositions of $\alpha$ and $\beta$ respectively. In this case $C(\nu, \rho)=c_{\nu, \rho}^{n}(g(\nu, \rho)=0)$.

\subsection{Ordered rooted bicolored thorn trees}

We define the following sets of trees:

Definition 2 ( Ordered rooted bicolored trees) For $\lambda, \mu \vdash n$ such that $\ell(\lambda)+\ell(\mu)=n+1$, let $\mathcal{B T}(\lambda, \mu)$ be the set of ordered rooted bicolored trees $t$ with $\ell(\lambda)$ white vertices of degree distribution given by $\lambda$, and $\ell(\mu)$ black vertices of degree distribution given by $\mu$. By convention, the root is a white vertex.

If we are only interested in the number of vertices, let $\mathcal{B T}(p, q)=\bigcup_{\ell(\lambda)=p, \ell(\mu)=q} \mathcal{B T}(\lambda, \mu)$. As shown e.g. in (2), the cardinality of $\mathcal{B T}(\lambda, \mu)$ when $\ell(\lambda)+\ell(\mu)=n+1$ is:

$$
|\mathcal{B T}(\lambda, \mu)|=\frac{n}{\operatorname{Aut}(\lambda) \operatorname{Aut}(\mu)}(n-\ell(\lambda)) !(n-\ell(\mu)) !
$$

and they are in bijection with the ordered factorizations counted by $c_{\lambda \mu}^{n}$ when $g(\lambda, \mu)=0$. By Remark 2. in this case we also have $C(\lambda, \mu)=c_{\lambda \mu}^{n}$. To get a combinatorial construction for $\mathcal{C}(\lambda, \mu)$ when $\ell(\lambda)+\ell(\mu)<n+1$ we introduce the ordered rooted bicolored thorn trees:

Definition 3 ( Ordered rooted bicolored thorn trees) We call a thorn an edge connected to only one vertex. For $\lambda, \mu \vdash n$ such that $\ell(\lambda)+\ell(\mu) \leq n+1$, we define $\widetilde{\mathcal{B T}}(\lambda, \mu)$ as the set of ordered rooted bicolored trees with $\ell(\lambda)$ white vertices, $\ell(\mu)$ black vertices, $n+1-\ell(\lambda)-\ell(\mu)$ thorns connected to the black vertices and $n+1-\ell(\lambda)-\ell(\mu)$ thorns connected to the white vertices. The white (respectively black) vertices' degree distribution (accounting the thorns) is specified by $\lambda$ (respectively $\mu$ ). The root is a white vertex. 
Adapting the Lagrange inversion developed in (2), it can be shown that:

$$
\widetilde{\mathcal{B \mathcal { T }}}(\lambda, \mu) \mid=\frac{n}{\operatorname{Aut}(\lambda) \operatorname{Aut}(\mu)} \frac{(n-\ell(\lambda)) !(n-\ell(\mu)) !}{(n+1-\ell(\lambda)-\ell(\mu)) !^{2}}
$$

Example 2 The tree on Figure 2 belongs to $\widetilde{\mathcal{B T}}(\lambda, \mu)$ for $n=11, \lambda=\left[1^{2}, 2,3,4\right]$ and $\mu=\left[1,2,4^{2}\right]$ $(\ell(\lambda)+\ell(\mu)=9<11)$.

Remark 3 One can notice the central role of the quantity $n+1-\ell(\lambda)-\ell(\mu)(=2 g(\lambda, \mu)$ in the case of $\alpha \in \mathcal{C}_{\lambda}$ and $\alpha^{-1} \gamma_{n} \in \mathcal{C}_{\mu}$, i.e. maps).

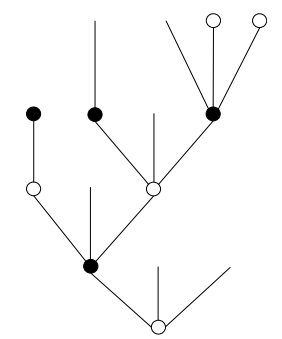

Fig. 2: An ordered rooted bicolored thorn Tree

\subsection{New formulation of the main theorem}

By equation (3), Theorem 1 is equivalent to proving the following formula:

$C(\lambda, \mu)=\frac{n}{\operatorname{Aut}(\lambda) \operatorname{Aut}(\mu)} \frac{(n-\ell(\lambda)) !(n-\ell(\mu)) !}{(n+1-\ell(\lambda)-\ell(\mu)) !^{2}} \times(n+1-\ell(\lambda)-\ell(\mu)) !=|\widetilde{\mathcal{B T}}(\lambda, \mu)| \times \mid S_{n+1-\ell(\lambda)-\ell(\mu)}$.

In this paper we show this formula bijectively:

Theorem 2 There is a direct bijection between partitioned bicolored maps $\mathcal{C}(\lambda, \mu)$ and pairs $(\tau, \sigma)$ of a bicolored thorn tree $\tau \in \widehat{\mathcal{B T}}(\lambda, \mu)$ and a permutation $\sigma \in S_{n+1-\ell(\lambda)-\ell(\mu)}$.

\section{A mapping $\Psi$ for bicolored partitioned maps of specified type}

To prove Theorem 2, we first need to define some additional structures.

\subsection{Reverse levels traversals of thorn trees and partial permutations}

Definition 4 (Reverse white and black levels traversals) For $\tau$ in $\widetilde{\mathcal{B T}}(\lambda, \mu)$, we define the reverse white levels traversal as the traversal going through all white vertices of $\tau$ and their descendants, either black vertices or thorns, in the following order (we assume that level 1 is the root's level):

(i) The descendants (either black vertices or thorns if any) of the leftmost white vertex of the top white level are traversed from left to right.

(ii) Then the leftmost white vertex of the top white level is traversed. 
(iii) If $i$ white vertices $(1, \ldots, i)$ (1 being the leftmost) of white level $j(j>1)$ have been traversed and there is a white vertex $i+1$ at level $j$ on the right of $i$, the descendants of $i+1$ are traversed, then vertex $i+1$ is traversed. Otherwise, the descendants of vertex 1 of white level $j-1$ are traversed, followed by the white vertex itself.

(iv) The root vertex is the last to be traversed.

The reverse black levels traversal is defined similarly except that the rightmost black descendant of the white root vertex is the last vertex to be traversed.
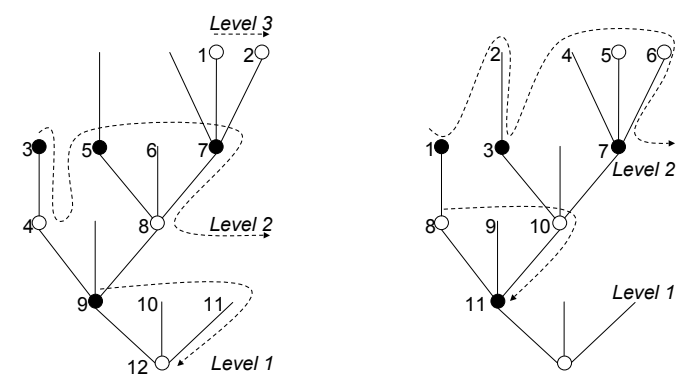

Fig. 3: Reverse white levels traversal (left) and reverse black levels traversal (right)

We also define partial permutations as in (9).

Definition 5 (Partial permutations) Given two sets $X$ and $Y$ and a nonnegative integer $m$, let $\mathcal{P} \mathcal{P}(X, Y, m)$ be the set of bijections from any $m$-subset of $X$ to any $m$-subset of $Y$ We call these bijections partial permutations. Then $|\mathcal{P} \mathcal{P}(X, Y, m)|=\left(\begin{array}{c}|X| \\ m\end{array}\right)\left(\begin{array}{c}|Y| \\ m\end{array}\right)$ m! Trivially, a partial permutation $\widetilde{\sigma} \in \mathcal{P} \mathcal{P}(X, Y, m)$ is also specified by a permutation $\sigma \in S_{m}$ and the complements of the domain and range.

We proceed with the description of the bijective mapping $\Psi$,

$$
\Psi_{n, \lambda, \mu}: \mathcal{C}(\lambda, \mu) \longrightarrow \widetilde{\mathcal{B T}}(\lambda, \mu) \times S_{n+1-\ell(\lambda)-\ell(\mu)}, \quad\left(\pi_{1}, \pi_{2}, \alpha\right) \longmapsto(\tau, \sigma) .
$$

\subsection{The ordered bicolored thorn tree $\tau$}

Let $\left(\pi_{1}, \pi_{2}, \alpha\right) \in \mathcal{C}(\lambda, \mu)$. We construct an ordered bicolored thorn tree $\tau \in \widetilde{\mathcal{B T}}(\lambda, \mu)$ following the procedure below (in what follows, $p=\ell(\lambda)$ and $q=\ell(\mu)$ ).

(i) First step is to construct the last passage unlabeled bicolor tree $t$ and two "relabeling" permutations $\theta_{1}$ and $\theta_{2}$ defined in the same way as in (9). We briefly describe this construction:

Let $\pi_{1}^{(1)}, \ldots, \pi_{1}^{(p)}$ and $\pi_{2}^{(1)}, \ldots, \pi_{2}^{(q)}$ be the blocks of the partitions $\pi_{1}$ and $\pi_{2}$ respectively. Denote by $m_{1}^{(i)}$ the maximal element of the block $\pi_{1}^{(i)}(1 \leq i \leq p)$ and by $m_{2}^{(j)}$ the maximal element of $\pi_{2}^{(j)}(1 \leq j \leq q)$. We assign the index $p$ to the block of $\pi_{1}$ containing the element 1 , and suppose that the indexing of all other blocks is arbitrary. We first construct the labeled ordered tree $T$ in the following way: The white root is labeled $p$. For every $j=1, \ldots, q$, the black vertex $j$ is a 
descendant of the white vertex $i$ if the element $\beta\left(m_{2}^{(j)}\right)$ belongs to the white block $\pi_{1}^{(i)}$. Similarly, for every $i=1, \ldots, p-1$, a white vertex $i$ is a descendant of a black vertex $j$ if the element $m_{1}^{(i)}$ belongs to the black block $\pi_{2}^{(j)}$. If black vertices $j, k$ are both descendants of a white vertex $i$, then $j$ is to the left of $k$ when $\beta\left(m_{2}^{(j)}\right)<\beta\left(m_{2}^{(k)}\right)$; if white vertices $i, l$ are both descendants of a black vertex $j$, then $i$ is to the left of $l$ when $\beta^{-1}\left(m_{1}^{(i)}\right)<\beta^{-1}\left(m_{1}^{(l)}\right)$. It is not hard to show that this construction gives a tree (9) that we denote by $T$.

We remove the labels to obtain the unlabeled bicolored ordered tree $t$. Relabeling permutations $\theta_{1}$ and $\theta_{2}$ are defined by considering the reverse-labelled tree $T^{\prime}$ resulting from the labelling of $t$, based on two independent reverse-labelling procedures for white and black vertices. The root is labelled $p$, then going bottom up and right to left, we label the white vertices with labels $p-1, p-2, \ldots 1$. An equivalent procedure applies to black vertices. Next step consists in relabeling the blocks by using the new indices from $T^{\prime}$. If a white vertex is labeled $i$ in $T$ and $i^{\prime}$ in $T^{\prime}$, we set $\pi_{1}^{i^{\prime}}=\pi_{1}^{(i)}$. Black blocks are relabeled in a similar fashion. Let $\omega^{i}, v^{j}$ be the strings given by writing the elements of $\pi_{1}^{i}, \pi_{2}^{j}$ in increasing order. Denote by $\omega=\omega^{1} \ldots \omega^{p}, v=v^{1} \ldots v^{q}$, the concatenations of the strings defined above. We define $\theta_{1} \in S_{n}$ by setting $\omega$ as the first line and $[n]$ as the second line of the two-line representation of this permutation. Similarly, we define the relabeling permutation $\theta_{2}$.

Example 3 Let $n=8, \lambda=\left[2^{4}\right], \mu=\left[2,3^{2}\right], \alpha=(23)(4)(15)(6)(78), \beta=(2)(134)(568)(7)$, $\pi_{1}=\left\{\pi_{1}^{(1)}, \pi_{1}^{(2)}, \pi_{1}^{(3)}, \pi_{1}^{(4)}\right\}$ and $\pi_{2}=\left\{\pi_{2}^{(1)}, \pi_{2}^{(2)}, \pi_{2}^{(3)}\right\}$ with

$$
\begin{aligned}
& \pi_{1}^{(1)}=\{2,3\}, \quad \pi_{1}^{(2)}=\{4,6\}, \quad \pi_{1}^{(3)}=\{7,8\}, \quad \pi_{1}^{(4)}=\{1,5\}, \\
& \pi_{2}^{(1)}=\{1,3,4\}, \quad \pi_{2}^{(2)}=\{2,7\}, \quad \pi_{2}^{(3)}=\{5,6,8\}
\end{aligned}
$$

We associate shapes to the blocks as in Figure 4 and construct $T$.

We have that $\beta\left(m_{2}^{(2)}\right)=7 \in \pi_{1}^{(3)}, \beta\left(m_{2}^{(1)}\right)=1 \in \pi_{1}^{(4)}, \beta\left(m_{2}^{(3)}\right)=5 \in \pi_{1}^{(4)}$, and $\beta\left(m_{2}^{(1)}\right)<$ $\beta\left(m_{2}^{(3)}\right)$. Thus, the black vertex 2 is a descendant of the white vertex 3 , and the descendants, from left to right, of the white vertex 4 are the black vertices 1 and 3 . Also, we have that $m_{1}^{(1)}=3 \in \pi_{2}^{(1)}$, $m_{1}^{(2)}=6 \in \pi_{2}^{(3)}, m_{1}^{(3)}=8 \in \pi_{2}^{(3)}$ and $\beta^{-1}\left(m_{1}^{(2)}\right)<\beta^{-1}\left(m_{1}^{(3)}\right)$. Therefore, the white vertex 1 is a descendant of the black vertex 1 , and the descendants, from left to right, of the black vertex 3 are the white vertices 2 and 3. Finally, we associate shapes to the blocks as in Figure 4 Then, by removing the labels we get the tree $t$. Reverse labeling of t gives $T^{\prime}$ and consequently the relabeling permutations:

$$
\theta_{1}=\left(\begin{array}{ll|ll|ll|ll}
2 & 3 & 4 & 6 & 7 & 8 & 1 & 5 \\
1 & 2 & 3 & 4 & 5 & 6 & 7 & 8
\end{array}\right) \quad \theta_{2}=\left(\begin{array}{ll|lll|lll}
2 & 7 & 1 & 3 & 4 & 5 & 6 & 8 \\
1 & 2 & 3 & 4 & 5 & 6 & 7 & 8
\end{array}\right)
$$

(ii) We use the sets

$$
\left\{\theta_{1}\left(m_{1}^{i}\right)\right\}_{1 \leq i \leq p-1} \cup\left\{\theta_{1}\left(\beta\left(m_{2}^{j}\right)\right)\right\}_{1 \leq j \leq q} \text { and }\left\{\theta_{2}\left(\beta^{-1}\left(m_{1}^{i}\right)\right)\right\}_{1 \leq i \leq p-1} \cup\left\{\theta_{2}\left(m_{2}^{j}\right)\right\}_{1 \leq j \leq q}
$$




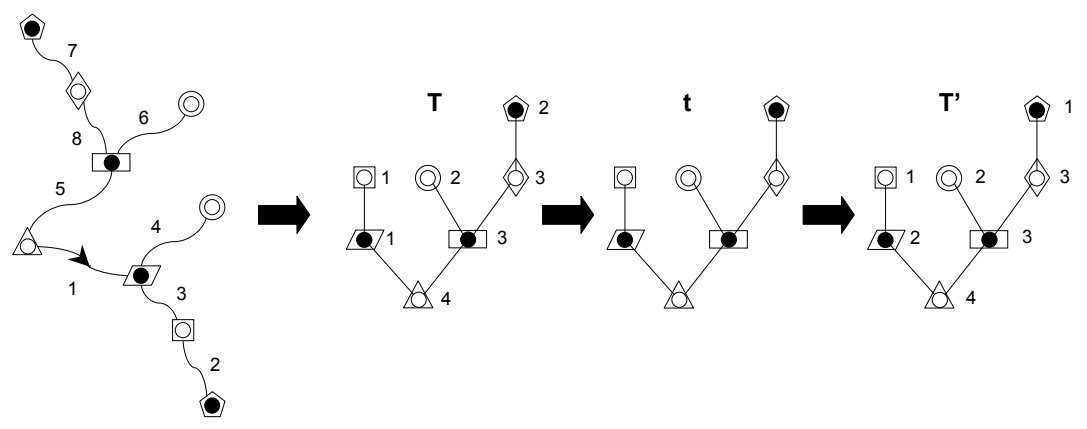

Fig. 4: Construction of $\mathrm{T}, \mathrm{t}$ and $\mathrm{T}$ '

in order to double-label tree $t$. We assign $\left(\theta_{1}\left(m_{1}^{i}\right), \theta_{2}\left(\beta^{-1}\left(m_{1}^{i}\right)\right)\right)$ to the white vertex indexed by $i,(i<p)$ in $T^{\prime}$ and $\left(\theta_{1}\left(\beta\left(m_{2}^{j}\right)\right), \theta_{2}\left(m_{2}^{j}\right)\right)$ to black vertex indexed by $j$ in $T^{\prime}$. The root vertex is first labeled $n=\theta_{1}\left(m_{1}^{p}\right)$ and has no second label. We call $T^{\prime \prime}$ the resulting double-labeled tree (see figure 5 for an example).

Let $S_{0}=\left\{m_{1}^{1}, \ldots, m_{1}^{p-1}\right\} \cup\left\{\beta\left(m_{2}^{1}\right), \ldots, \beta\left(m_{2}^{q}\right)\right\}$ and $\overline{\theta_{1}\left(S_{0}\right)}$ (resp. $\overline{\theta_{2}\left(\beta^{-1}\left(S_{0}\right)\right)}$ ) be the ordered subset of $[n]$ obtained by arranging the elements of $\theta_{1}\left(S_{0}\right)$ (resp. $\theta_{2}\left(\beta^{-1}\left(S_{0}\right)\right)$ ) in increasing order. We have the following lemmas:

Lemma 1 ((9)) The set $S_{0}$ has $p+q-1$ elements, i.e. $\left\{m_{1}^{1}, \ldots, m_{1}^{p-1}\right\} \cap\left\{\beta\left(m_{2}^{1}, \ldots, \beta\left(m_{2}^{q}\right)\right\}=\emptyset\right.$.

Lemma 2 Let $d=\left(d_{1}, d_{2}, \ldots, d_{p+q-1}\right)$ (resp. $d^{\prime}=\left(d_{1}^{\prime}, d_{2}^{\prime}, \ldots, d_{p+q-1}^{\prime}\right)$ ) be the ordered set of first (resp. second) labels obtained by traversing $T^{\prime \prime}$ up to, but not including, the root vertex according to the reverse white (resp. black) levels traversal defined in section 3 . We have :

$$
d=\overline{\theta_{1}\left(S_{0}\right)}, \quad \text { and } \quad d^{\prime}=\overline{\theta_{2}\left(\beta^{-1}\left(S_{0}\right)\right)} .
$$

Proof: Let $\theta_{1}\left(m_{1}^{0}\right)=0$. According to our construction, if a black vertex with first label $\theta_{1}\left(\beta\left(m_{2}^{j}\right)\right)$ in $T^{\prime \prime}$ is a descendant of a white vertex with first label $\theta_{1}\left(m_{1}^{i}\right),(1 \leq i \leq p)$, then $\beta\left(m_{2}^{j}\right)$ belongs to $\pi_{1}^{i}$ and $\beta\left(m_{2}^{j}\right) \leq m_{1}^{i}$. As the image by $\theta_{1}$ of any element of the white blocks $\left\{\pi_{1}^{l}\right\}_{1 \leq l<i}$ is strictly less than the image by $\theta_{1}$ of any element of $\pi_{1}^{i}$, and $\theta_{1}$ is an increasing function on each block $\left\{\pi_{1}^{i}\right\}_{1 \leq i \leq p}$ we have:

$$
\theta_{1}\left(m_{1}^{i-1}\right)<\theta_{1}\left(\beta\left(m_{2}^{j}\right)\right)<\theta_{1}\left(m_{1}^{i}\right), \text { where } \theta_{1}\left(m_{1}^{0}\right)=0 .
$$

Suppose now that black vertices $j$ and $k$ with first labels $\theta_{1}\left(\beta\left(m_{2}^{j}\right)\right)$ and $\theta_{1}\left(\beta\left(m_{2}^{k}\right)\right)$ in $T^{\prime \prime}$ are both descendants of the same white vertex and that $j$ is on the left of $k$. The construction of $t$ and $T^{\prime \prime}$ implies that $\beta\left(m_{2}^{j}\right)<\beta\left(m_{2}^{k}\right)$. As $\theta_{1}$ is increasing on the white blocks then $\theta_{1}\left(\beta\left(m_{2}^{j}\right)\right)<$ $\theta_{1}\left(\beta\left(m_{2}^{k}\right)\right)$. Finally, reverse white levels traversal of the first labels in $T^{\prime \prime}$ (up to but not including the root) yields $\overline{\theta_{1}\left(S_{0}\right)}$. Similarly, reverse black levels traversal of the second labels in $T^{\prime \prime}$ yields $\overline{\theta_{2}\left(\beta^{-1}\left(S_{0}\right)\right)}$. 
(iii) We add $n+1-p-q$ thorns to the white vertices and also $n+1-p-q$ thorns to the black vertices in $T^{\prime \prime}$ in the following fashion. Let $d$ and $d^{\prime}$ be defined as in Lemma 2. We know from Lemma 1 and 2 that $d$ and $d^{\prime}$ are strictly increasing subsequence of $[n]$ obtained by reverse levels traversals of $T^{\prime \prime}$. First, we add thorns to the white vertices in the following way:

- If $d_{1}>1$ and the vertex with first label $d_{1}$ is white, we connect $d_{1}-1$ thorns to it. If the vertex with first label $d_{1}$ is black, we connect $d_{1}-1$ thorns on the left of the ascending white vertex.

- For $1<l<p+q-1$, if $d_{l}>d_{l-1}+1$ we follow exactly one of the four following cases: If $d_{l}$ and $d_{l-1}$ are both the first label of white vertices in $T^{\prime \prime}$, white vertex $d_{l}$ (short for vertex corresponding to $d_{l}$ ) has no black descendant and it is the white vertex following $d_{l-1}$ in the reverse white levels traversal of $T^{\prime \prime}$. We connect $d_{l}-d_{l-1}-1$ thorns to vertex $d_{l}$.

If $d_{l}$ is the first label of a black vertex and $d_{l-1}$ is the first label of a white one, then black vertex $d_{l}$ is the leftmost descendant of the white vertex following $d_{l-1}$. We connect $d_{l}-d_{l-1}-1$ thorns on the left of the ascending white vertex of $d_{l}$.

If $d_{l}$ is the first label of a white vertex and $d_{l-1}$ is the first label of a black one, then the black vertex $d_{l-1}$ is the rightmost descendant of vertex $d_{l}$. We connect $d_{l}-d_{l-1}-1$ thorns to vertex $d_{l}$ on the right of vertex $d_{l-1}$

Finally, if $d_{l}$ and $d_{l-1}$ are both the first label of black vertices, these two vertices have the same white ascending vertex. We connect $d_{l}-d_{l-1}-1$ thorns to the ascending white vertex between these two black vertices.

- If $d_{p+q-1}<n$, we connect $n-d_{p+q-1}-1$ thorns to the root vertex on the right of its rightmost black descendant.

We can think of this as adding a thorn to the white vertices for each integer of $[n]$ not included in $d$. A similar construction is applied to add thorns to the black vertices following the sequence of integers $d^{\prime}$. Finally, we remove all the labels to get the ordered bicolored thorn tree $\tau$.

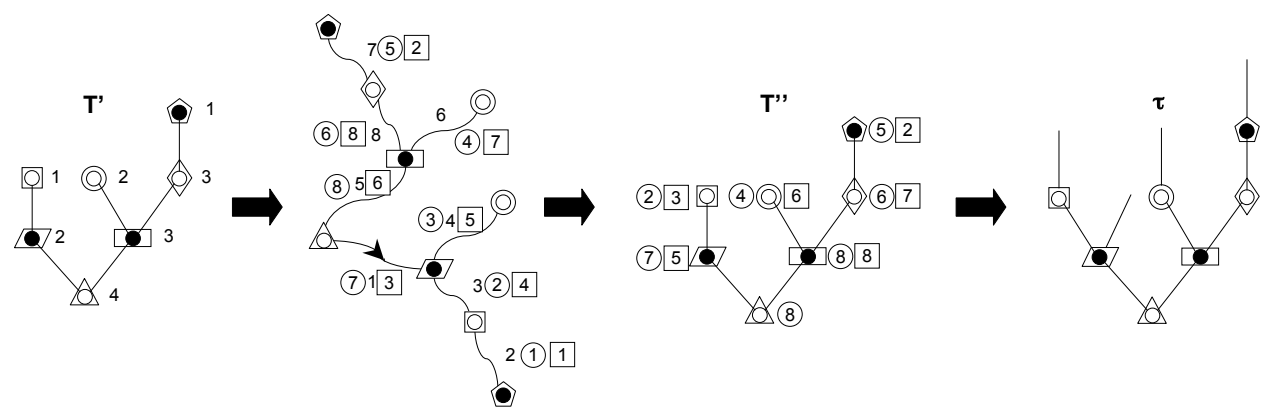

Fig. 5: Construction of $T^{\prime \prime}$ and $\tau$. Circled numbers correspond to the first relabeling permutation $\theta_{1}$ and the first labels in $T^{\prime \prime}$. Squared numbers correspond to the second relabeling permutation $\theta_{2}$ and the second labels in $T^{\prime \prime}$. 
Example 4 Following the previous example, the corresponding $T^{\prime \prime}$ and $\tau$ are represented on figure 5. In order to get $\tau$ from $T^{\prime \prime}$, we use $d=(2,4,5,6,7,8)$ and $d^{\prime}=(2,3,5,6,7,8)$. The missing integers in $d$ are 1 and 3 , so we connect one thorn to the white vertex with first label 2 (since $d_{1}>1$ ) and one to the white vertex with first label 4 ( since $\left.d_{2}>d_{1}+1\right)$. Similarly, we connect one thorn to the black vertex with second label $2\left(\right.$ since $\left.d_{1}^{\prime}>1\right)$ and one to the right of the black vertex with second label 5 (since $d_{3}^{\prime}>d_{2}^{\prime}+1$ ).

Lemma $3 \tau$ as defined above belongs to $\widetilde{\mathcal{B T}}(\lambda, \mu)$

Proof: As there are $p+q-1$ distinct elements in $d$ and $d^{\prime}$, exactly $n-|d|=n+1-p-q$ thorns are connected to the white vertices. Similarly $n+1-p-q$ thorns are connected to the black vertices. Then if we take two successive white vertices $i-1$ and $i$ according to the reverse white level traversal of $T^{\prime \prime}$ with first labels $\theta_{1}\left(m_{1}^{i-1}\right)$ and $\theta_{1}\left(m_{1}^{i}\right),(i<p)$, a thorn is connected to $i$ for each integer of $\left[\theta_{1}\left(m_{1}^{i-1}\right), \theta_{1}\left(m_{1}^{i}\right)\right]$ missing in $d$. The number of these missing integers is equal to $\theta_{1}\left(m_{1}^{i}\right)-1-\theta_{1}\left(m_{1}^{i-1}\right)-f_{i}$ where $f_{i}$ is the number of black descendants of $i$. As $i$ is not the root vertex, there is an edge between $i$ and its ascendant so that the resulting degree $v$ for $i$ is:

$$
\forall i \in[p-1], v(i)=\theta_{1}\left(m_{1}^{i}\right)-1-\theta_{1}\left(m_{1}^{i-1}\right)-f_{i}+f_{i}+1=\theta_{1}\left(m_{1}^{i}\right)-\theta_{1}\left(m_{1}^{i-1}\right)
$$

Furthermore, $n-\theta_{1}\left(m_{1}^{p-1}\right)-f_{p}$ thorns are connected to the root vertex so that:

$$
v(p)=n-\theta_{1}\left(m_{1}^{p-1}\right)
$$

But, according to the construction of $\theta_{1}$,

$$
\begin{aligned}
& \theta_{1}\left(\pi_{1}^{1}\right)=\left[\theta_{1}\left(m_{1}^{1}\right)\right] \\
& \theta_{1}\left(\pi_{1}^{i}\right)=\left[\theta_{1}\left(m_{1}^{i}\right)\right] \backslash\left[\theta_{1}\left(m_{1}^{i-1}\right)\right],(2 \leq i \leq p-1) \\
& \theta_{1}\left(\pi_{1}^{p}\right)=[n] \backslash\left[\theta_{1}\left(m_{1}^{p-1}\right)\right]
\end{aligned}
$$

Subsequently for $i \in[1, p], v(i)=\left|\pi_{1}^{i}\right|$, and $\lambda=\operatorname{type}(\pi)$ is the white vertex degree distribution of $\tau$. In a similar fashion, $\mu$ is the black vertex degree distribution of $\tau$.

Lemma 4 Assign a first set of labels $1,2, \ldots, n$ to the vertices and the thorns connected to white vertices in $\tau$ in increasing order according to the reverse white levels traversal as well as a second set of labels $1,2, \ldots, n$ to the vertices and the thorns connected to black vertices in increasing order according to the reverse black levels traversal. The double-labeling of the vertices is the same as in $T^{\prime \prime}$.

Proof: According to the construction of $\tau$, we add thorns to $T^{\prime \prime}$ when integers are missing in the reverse levels traversals of $T^{\prime \prime}$ so that the thorns would take these missing integers as labels when traversing the thorn tree. As a result, the labels of the vertices in the reverse levels traversals of $\tau$ are still $d$ and $d^{\prime}$ and since they still appear in the same order, we have the desired result. 


\subsection{The permutation $\sigma$}

Let $S=[n] \backslash S_{0}$, we define the partial permutation $\widetilde{\sigma}$ on set $\theta_{1}(S)$ by:

$$
\widetilde{\sigma}=\left.\theta_{2} \circ \beta^{-1} \circ \theta_{1}^{-1}\right|_{\theta_{1}(S)} .
$$

Then let $\sigma$ be the permutation in $S_{n+1-p-q}$ that is order-isomorphic to $\widetilde{\sigma}$. That is, we define the ordered set $\overline{\theta_{1}(S)}$ with all the elements of $\theta_{1}(S)$ sorted in increasing order and $\rho_{1}$ the labeling function which associates to each element of $\theta_{1}(S)$ its position index in $\overline{\theta_{1}(S)}$. As a direct consequence, we have $\rho_{1}\left(\theta_{1}(S)\right)=[n+1-p-q]$. Similarly we define $\rho_{2}$ to label the elements of $\theta_{2}\left(\beta^{-1}(S)\right)$. Then $\sigma$ is given by:

$$
\sigma:[n+1-p-q] \longrightarrow[n+1-p-q], \quad u \longmapsto \rho_{2} \circ \tilde{\sigma} \circ \rho_{1}^{-1}(u)
$$

Example 5 Going ahead with our previous example, we find:

$$
\widetilde{\sigma}=\left(\begin{array}{ll}
1 & 3 \\
1 & 4
\end{array}\right) \quad \text { and } \quad \sigma=\left(\begin{array}{ll}
1 & 2 \\
1 & 2
\end{array}\right) .
$$

\section{Proof that the mapping $\Psi$ is a bijection}

Theorem $3 \Psi_{n, \lambda, \mu}$ is actually a bijection

Proof: To show that $\Psi_{n, \lambda, \mu}$ is a one-to-one correspondence we take any element $(\tau, \sigma)$ in $\widetilde{\mathcal{B T}}(\lambda, \mu) \times$ $S_{n+1-\ell(\lambda)-\ell(\mu)}$ and show that there is a unique element $\left(\pi_{1}, \pi_{2}, \alpha\right)$ in $\mathcal{C}(\lambda, \mu)$ such that $\Psi_{n, \lambda, \mu}\left(\pi_{1}, \pi_{2}, \alpha\right)=(\tau, \sigma)$. Let $p=\ell(\lambda)$ and $q=\ell(\mu)$. We proceed with a two step proof:

(i) The first step is to notice that $(\tau, \sigma)$ defines a unique unlabeled bicolored tree $t$ and a unique partial permutation $\widetilde{\sigma}$ belonging to $\mathcal{B T}(p, q) \times \mathcal{P} \mathcal{P}(n, n-1, n+1-p-q)$. Double-labeling of $\tau$ with $1,2, \ldots, n$ in increasing order according to the reverse levels traversals and removing the two sets of $n+1-p-q$ thorns (together with their labels) gives a double-labeled tree $T^{\prime \prime}$ that leads to $\tau$ according to $\Psi$. This double-labeled tree is the unique one that can lead to $\tau$ since within $\Psi$, the constructions of $\tau$ and $T^{\prime \prime}$ have the same underlying tree structure, and according to Lemma $4, \tau$ determines the labels of $T^{\prime \prime}$.

Then, using Lemma2, the two series of labels (except the root's) in $T^{\prime \prime}$ are necessarily the missing elements in the domain (first labels) and the range (second labels) of $\widetilde{\sigma}$ sorted in increasing order (within the reverse levels traversals). Hence, $T^{\prime \prime}$ and $\sigma$ uniquely determine $t$ and $\widetilde{\sigma}$. Obviously, the process of using the labels in $T^{\prime \prime}$ as missing elements to reconstruct $\widetilde{\sigma}$ can always be performed. Within this process we have $\widetilde{\sigma}$ in $\mathcal{P} \mathcal{P}(n, n-1, n+1-p-q)$ since two series of $p+q-1$ labels are used and the second label of the last black vertex traversed in the reverse black levels traversal of $\tau$ is $n$ (since there are no other black vertices and no thorns connected to further black vertices either) and $n$ is always a missing element of the image.

(ii) The bijection $\Theta_{n, p, q}$ in (9) is identical to the first steps (up to the construction of $t$ and $\widetilde{\sigma}$ ) of $\Psi_{n, \lambda, \mu}$. There is therefore a unique triple $\left(\pi_{1}, \pi_{2}, \alpha\right)$ in $\mathcal{C}(p, q, n)=\bigcup_{\ell(\lambda)=p, \ell(\mu)=q} \mathcal{C}(\lambda, \mu)$ mapped to $t$ and $\widetilde{\sigma}$ by $\Theta$ and equivalently by the first steps of $\Psi$. But, according to (9), the types of $\pi_{1}$ and $\pi_{2}$ can be recovered with the pair $(t, \widetilde{\sigma})$ via the missing elements in the domain and range of $\widetilde{\sigma}$ corresponding to the relabeling by $\theta_{1}$ and $\theta_{2}$ of the maximum elements of the blocks (that we can 
identify by assigning the missing elements in $t$ using the reverse levels traversals). Furthermore, using Lemma 3, the vertex degree distribution of $\tau$ is equal to the type of the partitions encoded by the missing elements in $\widetilde{\sigma}$ corresponding to the relabeling of the maximum elements of the blocks. Finally, as the vertex degree distribution in $\tau$ is $(\lambda, \mu)$, so is the type of $\left(\pi_{1}, \pi_{2}\right)$; obtained as part of the preimage of $(t, \widetilde{\sigma})$ under $\Theta$. Therefore, $\left(\pi_{1}, \pi_{2}, \alpha\right)$ belongs to $\mathcal{C}(\lambda, \mu)$ as desired.

\section{Acknowledgements}

The authors are grateful to Gilles Schaeffer for drawing their attention to the problem.

\section{References}

[1] F. Bédard and A. Goupil The poset of conjugacy classes and decomposition of products in the symmetric group. Can. Math. Bull, 35(2):152-160, 1992.

[2] I. P. Goulden and D. M. Jackson. The Combinatorial Relationship Between Trees, Cacti and Certain Connection Coefficients for the Symmetric Group. European Journal of Combinatorics, 13:357-365, 1992.

[3] A. Goupil A. and G. Schaeffer. Factoring n-cycles and counting maps of given genus. European Journal of Combinatorics, 19:819-834(16), 1998.

[4] D. Poulalhon and G. Schaeffer. Factorizations of large cycles in the symmetric group. Discrete Math., 254:433-458, 2000.

[5] P. Biane. Nombre de factorisations d'un grand cycle. Séminaire Lotharingien de Combinatoire, 51:4, 2004.

[6] I. P. Goulden and A. Nica A direct bijection for the Harer-Zagier formula. J. Comb. Theory Ser. A, 111(2):224-238, 2005.

[7] J. Irving. On the number of factorizations of a full cycle. J. Comb. Theory Ser. A, 113(7):1549-1554, 2006.

[8] D. M. Jackson. Some combinatorial problems associated with products of conjugacy classes of the symmetric group. J. Comb. Theory Ser. A, 49(2):363-369, 1988.

[9] G. Schaeffer and E. Vassilieva. A bijective proof of Jackson's formula for the number of factorizations of a cycle. J. Comb. Theory Ser. A, 115(6):903-924, 2008.

[10] R. P. Stanley. Enumerative Combinatorics, volume 2. Cambridge University Press, Cambridge, 1999.

[11] G. M. Ziegler. On the poset of partitions of integers. j. Comb. Theory Ser. A, 42(2):215-222, 1986. 